\title{
The fate of axial U(1) in $2+1$ flavor QCD towards the chiral limit
}

\section{Lukas Mazur*}

Fakultät für Physik, Universität Bielefeld, D-33615 Bielefeld, Germany

E-mail: lmazur@physik.uni-bielefeld.de

\section{Olaf Kaczmarek}

Fakultät für Physik, Universität Bielefeld, D-33615 Bielefeld, Germany

E-mail: okacz@physik.uni-bielefeld.de

\section{Edwin Laermann ${ }^{\dagger}$}

Fakultät für Physik, Universität Bielefeld, D-33615 Bielefeld, Germany

\section{Sayantan Sharma}

The Institute of Mathematical Sciences, Chennai 600113, India

E-mail: sayantans@imsc.res.in

\begin{abstract}
The region of the Columbia plot with two light quark flavors is not yet conclusively understood. Non-perturbative effects, e.g. the magnitude of the anomalous U(1) axial symmetry breaking, decides on the nature of the phase transition in this region. We report on our study of this region of the Columbia plot using lattice techniques. We use gauge field ensembles generated within the Highly Improved Staggered Quark discretization scheme, with the strange quark mass fixed at its physical value and the light quark mass varied such that $m_{l}=m_{s} / 27$ and $m_{s} / 40$, where $m_{l}=m_{s} / 27$ corresponds to the physical light quark mass. We study the eigenvalue spectrum of QCD using the overlap Dirac operator on these gauge field ensembles at finite temperature around the chiral transition temperature $T_{c}$, as the light quark masses approach the chiral limit, and infer about the fate of the anomalous $U_{A}(1)$ symmetry breaking.
\end{abstract}

The 36th Annual International Symposium on Lattice Field Theory - LATTICE2018

22-28 July, 2018

Michigan State University, East Lansing, Michigan, USA.

\footnotetext{
${ }^{*}$ Speaker.

${ }^{\dagger}$ This work is dedicated to his loving memory
} 


\section{The $U_{A}(1)$ puzzle in QCD}

Symmetries determine the order parameters of a phase transition. The anomalous $U_{A}(1)$, though not an exact symmetry, is believed to affect the nature of the phase transition in two flavor QCD [1]. If $U_{A}(1)$ is indeed broken then the chiral phase transition for $N_{f}=2$ QCD is expected to be in the $O(4)$ universality class. Whether or not $U_{A}(1)$ is effectively restored at the chiral phase transition can only be answered non-perturbatively. Lattice studies of $N_{f}=2$ QCD have tried to address this problem. For latest updates see Ref. [2] and the talks in this conference [3,4].

We follow a different approach. Since the up and down quark masses are light compared to the intrinsic scale of $\mathrm{QCD}$, the $U_{L}(2) \times U_{R}(2)$ symmetry is only mildly broken. Therefore, if we calculate observables that measure the $U_{A}(1)$ breaking in $(2+1)$ flavor QCD with physical $u, d$ quark masses near the chiral crossover transition and reduce $m_{u, d}$, we can smoothly reach the chiral limit. If indeed $U_{A}(1)$ is broken, signatures of the $O(4)$ second order line could be observed by reducing $m_{u, d}$. On the other hand if $U_{A}(1)$ is effectively restored we should approach the $Z(2)$ line or a second order line of $U_{L}(2) \times U_{R}(2) / U_{V}(2)$ universality class [5]. In order to verify this we study in detail the eigenvalue spectrum of $(2+1)$ QCD with physical $m_{u, d}$ and compare the changes to it as the light quark mass is reduced successively. We then study its effects on $U_{A}(1)$ breaking observables as a function of light quark mass near the chiral crossover transition.

\section{QCD Dirac spectrum and $U_{A}(1)$ breaking}

Unlike chiral symmetry, it is not possible to use expectation values of local operators in the case of $U_{A}(1)$ to investigate its effective breaking or restoration as a function of temperature. Instead, it is important to look at the degeneracy of the correlation functions of mesons of appropriate quantum numbers. From Fig. 1 it is evident that if $U_{A}(1)$ is effectively restored then the meson correlators along the vertical axes of the box should be degenerate. Accordingly it was suggested

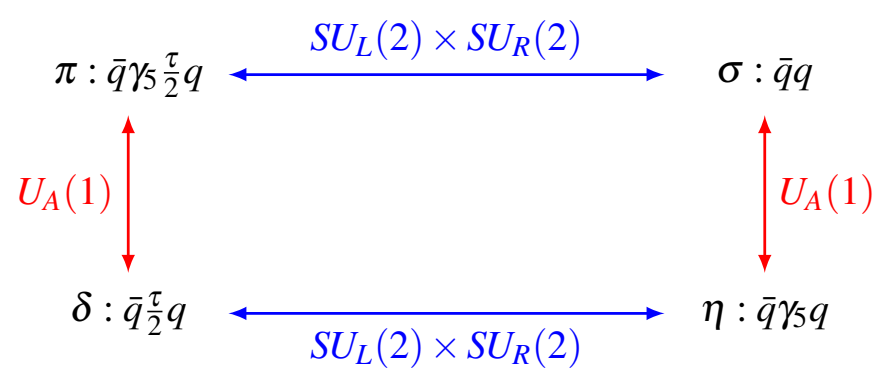

Figure 1: Symmetry transformations between scalar and pseudo-scalar mesons.

in Ref. [6] that the difference of the integrated correlators of pions and delta mesons, defined as $\chi_{\pi}-\chi_{\delta}=\int d^{4} x\left[\left\langle i \pi^{+}(x) i \pi^{-}(0)\right\rangle-\left\langle\delta^{+}(x) \delta^{-}(0)\right\rangle\right]$, is an observable that will quantify the amount of $U_{A}(1)$ breaking. This observable contains information about the microscopics of QCD since it can be written in terms of the eigenvalue density $\rho\left(\lambda, m_{l}\right)$ with the light quark mass $m_{l}=m_{u}=m_{d}$ of the QCD Dirac operator [7]

$$
\chi_{\pi}-\chi_{\delta} \stackrel{V \rightarrow \infty}{\longrightarrow} \int_{0}^{\infty} d \lambda \frac{4 m_{l}^{2} \rho\left(\lambda, m_{l}\right)}{\left(\lambda^{2}+m_{l}^{2}\right)^{2}} .
$$


In fact, from the study of up to 2-point correlation functions it has been shown [8,9] that when chiral symmetry is restored then the $U_{A}(1)$ can still be broken if the infrared part of eigenvalue density goes as $\lim _{\lambda \rightarrow 0} \rho\left(\lambda, m_{l}\right)=\delta(\lambda) m_{l}^{\alpha}$ with $1<\alpha<2$. It was further observed [10] that if the eigenvalue density is an analytic function in $m, \lambda$, then using chiral Ward Identities, it is possible to derive many useful properties of the eigenvalue spectrum of QCD at finite temperature in the chiral symmetry restored phase. From the study of up to 6-point correlation functions in the scalar and pseudo-scalar channels, a sufficient condition for the effective restoration of $U_{A}(1)$ in $N_{f}=2$ QCD is that the eigenvalue spectrum behaves as $\rho\left(\lambda, m_{l} \rightarrow 0\right) \sim \lambda^{3}$.

The properties of the eigenvalue density in QCD in the chiral symmetry restored phase can only be understood from non-perturbative lattice studies. Specifically, one has to calculate the small eigenvalues of the QCD Dirac operator on the lattice and verify that they have near-zero peak as well as parameterize the leading order analytic dependence of the spectrum. The existence of a small near-zero peak in the eigenvalue spectrum has been observed and were shown to be strongly sensitive to lattice cut-off effects [11]. Moreover, at high temperatures this near-zero peak arise due to weakly interacting instanton, anti-instanton pairs [12]. Hence, they are also sensitive to finite volume effects. However, the leading order analytic dependence of the infrared spectrum was observed to be quite robust, i.e. insensitive to lattice cut-off effects [13]. In fact, the leading analytic dependence of the eigenvalue spectrum changes from $\rho(\lambda) \sim \lambda$ at chiral crossover transition temperature $T_{c}$ to $\rho(\lambda) \sim \lambda^{2}$ at $1.2 T_{c}$ [12] for QCD with degenerate $u$ - $d$ quark masses slightly heavier than physical values. In these proceedings, we study the eigenvalue spectrum for physical quark masses and its changes as one approaches the chiral limit. We also to check if the near-zero peak survives in the chiral limit and how the eigenvalue spectrum influences the $U_{A}(1)$.

\section{Setup}

The gauge field ensembles used in this work were generated within the Highly Improved Staggered Quark (HISQ) discretization scheme with $2+1$ quark flavors. The strange quark mass was fixed at its physical value and the light quark mass was varied such that $m_{l}=m_{s} / 27$ and $m_{s} / 40$, where $m_{l}=m_{s} / 27$ corresponds to the physical quark mass. We used gauge field configurations separated by 100 Monte-Carlo trajectories. The details of the statistics and lattice sizes for the ensembles used for this study at each value of light quark mass and temperature are listed in table 1.

\begin{tabular}{|c|c|c|c|c|}
\hline$m_{s} / m_{l}$ & $N_{s}^{3} \times N_{\tau}$ & $\beta$ & $T / T_{c}$ & \#conf \\
\hline \hline 27 & $32^{3} \times 8$ & 6.390 & 0.97 & 69 \\
27 & $32^{3} \times 8$ & 6.445 & 1.03 & 81 \\
27 & $32^{3} \times 8$ & 6.500 & 1.09 & 102 \\
\hline \hline 40 & $32^{3} \times 8$ & 6.390 & 0.99 & 45 \\
40 & $32^{3} \times 8$ & 6.423 & 1.03 & 50 \\
40 & $32^{3} \times 8$ & 6.445 & 1.05 & 104 \\
\hline
\end{tabular}

Table 1: List of the configurations used in this work. 
Staggered fermions suffer from rooting problem and have no index theorem at finite lattice spacings. In fact the infrared part of the HISQ eigenvalue spectrum changes quite dramatically with changes in the lattice spacing, showing the emergence of a small near-zero peak at the finest lattice spacing $[14,15]$. Instead, we use the overlap Dirac operator to measure the low-lying eigenvalue spectrum for the HISQ ensembles. The overlap operator for one massless quark flavor is realized as

$$
D_{o v}=M\left[1+\gamma_{5} \operatorname{sgn}\left(\gamma_{5} D_{W}(-M)\right)\right],
$$

where $\operatorname{sgn}(\ldots)$ denotes the matrix sign function and $D_{W}$ is the massless Wilson-Dirac operator with a domain wall height $M \in[0,2)$. Since diagonalizing the overlap operator is computationally expensive, we first estimate the number and the chirality of the zero modes using the gluonic definition of the topological charge $Q=\int d^{4} x q(x)$, with the topological charge density defined as,

$$
q(x)=\frac{g^{2}}{32 \pi^{2}} \varepsilon_{\mu v \rho \sigma} \operatorname{tr}\left\{F_{\mu v}(x) F_{\rho \sigma}(x)\right\} .
$$

The gluonic definition of the topological charge is valid only for sufficiently smooth configurations. Thus, we have to apply a smoothing technique on the HISQ configurations to remove the ultraviolet fluctuations of the gauge fields before measuring the topological charge. In this work we use Wilson flow $[16,17]$ to measure $Q$. The flow smoothens the gauge fields over a region of radius $f=\sqrt{8 t}, t$ being the flow time. For each temperature we adjusted the flow time of the gauge fields such that $f T<1$ and the small instantons are not smoothened out.

For some configurations, the number and the chirality of zero modes measured using the Wilson flow was compared to the number of zero modes of the overlap Dirac operator. We found agreement in all the cases studied so far ensuring that the index theorem is valid. We used the information of the chirality of zero modes measured from the gluonic definition to construct a projected overlap operator $D_{o v}^{P}$ by projecting into the space of eigenvectors having the opposite chirality of the zero modes. This ensured that the projected overlap operator had no zero modes and was much faster to diagonalize on the lattice. The first 100 eigenvalues of $D_{o v}^{P \dagger} D_{o v}^{P}$ on the HISQ ensembles were calculated using the Kalkreuter-Simma (KS) Ritz algorithm [18]. Finally the eigenvectors of the overlap operator were computed using appropriate projections into the full vector-space.

Since the overlap operator was used to measure the eigenvalues of configurations generated with a different fermion discretization (HISQ) we renormalized the eigenvalue spectrum and the physical observables appropriately to eliminate the effects of this mixed action approach. In order to do so we calculated the valence (overlap) strange quark mass assuming that the ratio $m_{l} / m_{s}$ is fixed for both the valence and the sea sectors. We calculated an appropriate renormalized observable, $\Delta=\frac{m_{S}\langle\bar{\Psi} \Psi\rangle_{l}-m_{l}\langle\bar{\Psi} \Psi\rangle_{s}}{T^{4}}$, for the valence sector using the overlap eigenvalues and independently for the sea quark sector using the traces of derivatives and inverse of the HISQ operator using random noise vectors. The valence overlap mass $m_{s}$ was tuned such that these independent estimates of $\Delta$ are equal to each other.

\section{Our Results}

As mentioned earlier, we have chosen our configuration at every 100th Monte Carlo step to ensure that our results do not suffer from strong autocorrelations. To verify this, we plot the 
trajectory of the topological charge as a function of Monte Carlo time for each set of light quark masses and temperatures. Results are summarized in Fig. 2. From the plots it is evident that we have good sampling of the topological charge in our chosen set of gauge field ensembles. We
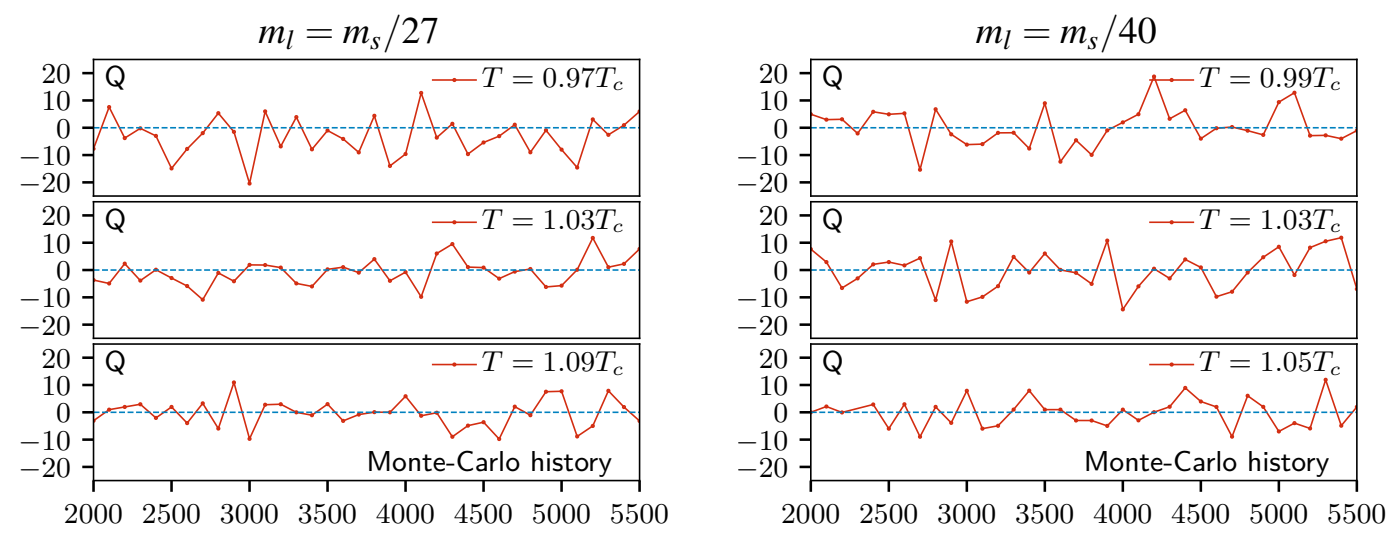

Figure 2: Trajectories of the topological charge of the lattices listed in table 1.

measured the eigenvalue spectrum using the overlap operator and renormalized the eigenvalues with the tuned valence strange quark mass $m_{s}$. As discussed earlier this was done such that the resultant density of the renormalized eigenvalues $\lambda / m_{s}$ is not sensitive to the mixed action approach artefacts. Our results for the QCD eigenvalue spectrum for two different values of light quark masses are summarized in Fig. 3. To understand the general features of the spectrum we fit it to the ansatz $\frac{\rho(\lambda)}{T^{3}}=\frac{\rho_{0} A}{A^{2}+\lambda^{2}}+c|\lambda|^{\gamma}$, where the terms in the R.H.S model the near-zero peak and the leading order analytic dependence of the eigenvalue spectra respectively. For the physical quark masses, the leading order analytic dependence of the eigenvalue density shown in the left panel of Fig. 3 goes as a power law with the exponent $\gamma \sim 1$ within errors for all the three temperatures studied. There is a small infrared peak (near-zero peak) whose relative contribution is suppressed with increasing temperatures. Lowering the sea light quark mass to $m_{s} / 40$, the general features of the eigenvalue spectrum remain unchanged as evident from the right panel of Fig. 3. The near-zero peak still survives and a linear analytic dependence on $\lambda$ is seen as expected from the predictions of chiral perturbation theory. The $m_{s} / 40$ results are still preliminary and the finite volume effects are under investigation.
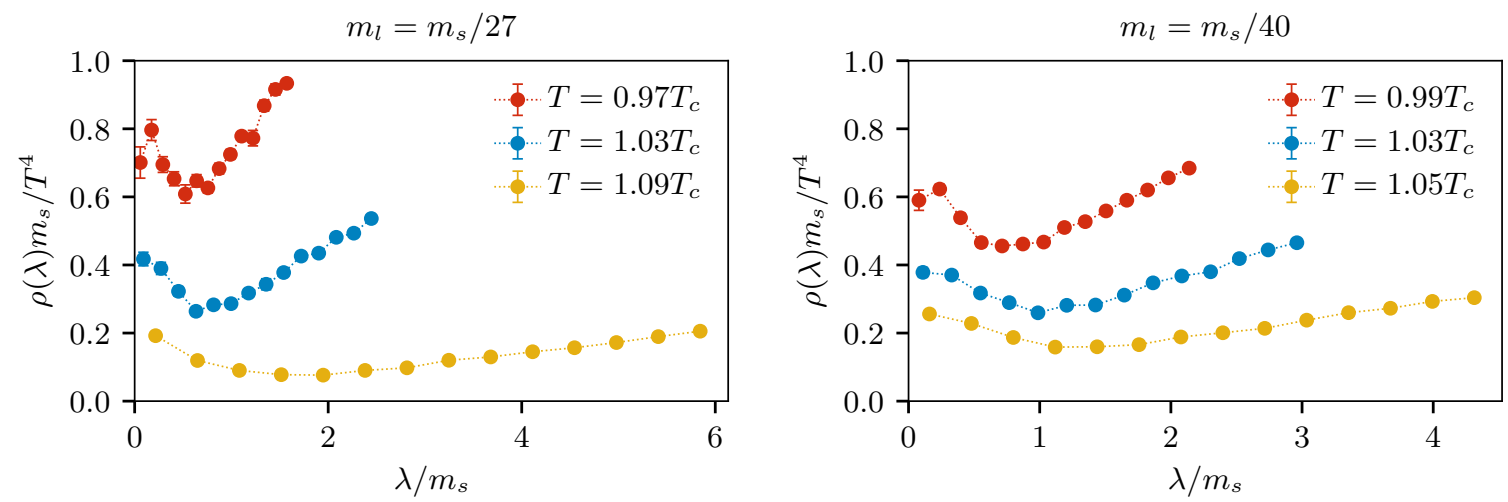

Figure 3: The eigenvalue density $\rho(\lambda)$ normalized to the tuned valence strange quark mass $m_{s}$. 
In order to study the $U_{A}(1)$ breaking as a function of temperature we calculated the renormalized observable $m_{s}^{2}\left(\chi_{\pi}-\chi_{\delta}\right) / T^{4}$, in terms of the scaled eigenvalues, which are summarized in Fig. 4. The red and blue points are for lines of constant physics corresponding to $m_{s} / m_{l}=27$ and $m_{s} / m_{l}=40$ respectively. The magnitude of $U_{A}(1)$ breaking at $T \sim T_{c}$ reduces with the quark mass, but it is still non-zero for $m_{l}=m_{s} / 40$, which in $\overline{\mathrm{MS}}$ scale is $\sim 2 \mathrm{MeV}$. This is in contrast to the results reported in [3], which observes a sudden fall-off of this observable to zero for $m_{l} \lesssim m_{l}^{\text {phys }}$. At present we have only two data points for this observable at each temperature, which prevents us from performing a proper chiral extrapolation.

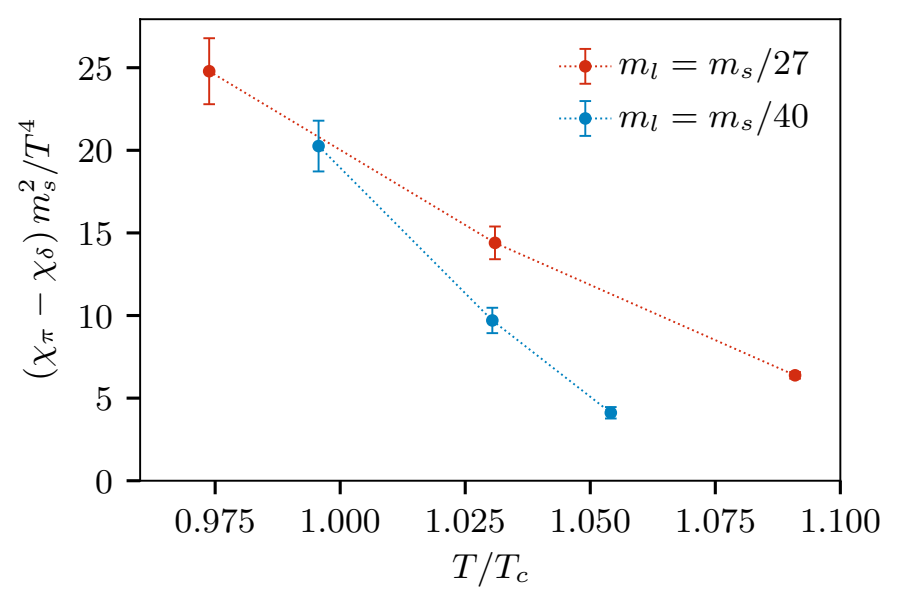

Figure 4: The renormalized $U_{A}(1)$ breaking parameter as a function of quark mass.

\section{Conclusions}

In this work we have studied the eigenvalue spectrum of the QCD Dirac operator near $T_{c}$ with physical value of strange quark mass and the light quark mass changed from its physical value $m_{s} / 27$ towards the chiral limit with $m_{l}=m_{s} / 40$. We do not find any drastic change in the features of the eigenvalue spectrum as a function of the light quark mass. The near-zero peak at the infrared part of the spectrum survives and the leading analytic dependence of the spectrum is linear in $\lambda$. Since we used overlap valence operators to measure the eigenvalue spectrum of the HISQ sea quarks, we appropriately renormalized the observables to reduce mixed action effects. We have further observed that the renormalized observable $m_{s}^{2}\left(\chi_{\pi}-\chi_{\delta}\right) / T^{4}$ is still non-zero even for $m_{l}=m_{s} / 40$ at $T \sim 1.1 T_{c}$, which shows $U_{A}(1)$ remains broken. We are currently measuring the eigenvalue spectrum and the $U_{A}(1)$ breaking at $m_{l}=m_{s} / 80$ which will eventually allow us to perform a proper chiral extrapolation and understand the $U_{A}(1)$ puzzle in the chiral limit.

\section{Acknowledgements}

The authors acknowledge support by the Deutsche Forschungsgemeinschaft (DFG) through grant CRC-TR 211, "Strong-interaction matter under extreme conditions". The computations were performed on the Bielefeld GPU cluster and we acknowledge PRACE for awarding us access to Piz Daint at CSCS, Switzerland. The GPU codes used in our work were in part based on some publicly available QUDA libraries [19]. 


\section{References}

[1] R. D. Pisarski and F. Wilczek, Remarks on the chiral phase transition in chromodynamics, Phys. Rev. D 29 (1984) 338.

[2] A. Tomiya, G. Cossu, S. Aoki, H. Fukaya, S. Hashimoto, T. Kaneko et al., Evidence of effective axial U(1) symmetry restoration at high temperature QCD, Phys. Rev. D96 (2017) 034509 [1612 . 01908].

[3] K. Suzuki et al., Axial U(1) symmetry and Dirac spectra in high-temperature phase of $N_{f}=2$ lattice QCD, These Proceedings.

[4] Y. Aoki et al., Topological Susceptibility in $N_{f}=2 Q C D$ at Finite Temperature - Volume Study, These Proceedings .

[5] A. Pelissetto and E. Vicari, Relevance of the axial anomaly at the finite-temperature chiral transition in $Q C D$, Phys. Rev. D88 (2013) 105018 [1309.5446].

[6] E. V. Shuryak, Which chiral symmetry is restored in hot QCD?, Comments Nucl. Part. Phys. 21 (1994) 235 [hep-ph/9310253].

[7] T. Hatsuda, S. H. Lee and H. Shiomi, QCD sum rules, scattering length and the vector mesons in nuclear medium, Phys. Rev. C52 (1995) 3364 [nucl-th/ 9505005 ].

[8] HotQCD collaboration, A. Bazavov et al., Chiral transition and $U(1)_{A}$ symmetry restoration from lattice $Q C D$ using domain wall fermions, Phys. Rev. D86 (2012) 094503 [1205.3535].

[9] M. I. Buchoff et al., QCD chiral transition, $U(1)_{A}$ symmetry and the dirac spectrum using domain wall fermions, Phys. Rev. D89 (2014) 054514 [1309.4149].

[10] S. Aoki, H. Fukaya and Y. Taniguchi, Chiral symmetry restoration, eigenvalue density of Dirac operator and axial U(1) anomaly at finite temperature, Phys. Rev. D86 (2012) 114512 [1209.2061].

[11] JLQCD collaboration, G. Cossu, H. Fukaya, S. Hashimoto and A. Tomiya, Violation of chirality of the Möbius domain-wall Dirac operator from the eigenmodes, Phys. Rev. D93 (2016) 034507 [1510 . 07395].

[12] V. Dick, F. Karsch, E. Laermann, S. Mukherjee and S. Sharma, Microscopic origin of $U(1)_{A}$ symmetry violation in the high temperature phase of QCD, Phys. Rev. D91 (2015) 094504 [1502.06190].

[13] S. Sharma, V. Dick, F. Karsch, E. Laermann and S. Mukherjee, The topological structures in strongly coupled QGP with chiral fermions on the lattice, Nucl. Phys. A956 (2016) 793 [1602.02197].

[14] H. Ohno, U. M. Heller, F. Karsch and S. Mukherjee, $U(1)_{A}$ breaking at finite temperature from the Dirac spectrum with the dynamical HISQ action, PoS LATTICE2012 (2012) 095 [1211. 2591].

[15] НотQCD collaboration, S. Sharma, The fate of $U(1)_{A}$ and topological features of $Q C D$ at finite temperature, 2018, 1801.08500 , DOI.

[16] R. Narayanan and H. Neuberger, Infinite N phase transitions in continuum Wilson loop operators, JHEP 03 (2006) 064 [hep-th/0601210].

[17] M. Lüscher, Properties and uses of the Wilson flow in lattice QCD, JHEP 08 (2010) 071 [1006.4518].

[18] T. Kalkreuter and H. Simma, An Accelerated conjugate gradient algorithm to compute low lying eigenvalues: A Study for the Dirac operator in SU(2) lattice QCD, Comput. Phys. Commun. 93 (1996) 33 [hep-lat/9507023].

[19] M. A. Clark, R. Babich, K. Barros, R. C. Brower and C. Rebbi, Solving Lattice QCD systems of equations using mixed precision solvers on GPUs, Comput. Phys. Commun. 181 (2010) 1517 [0911.3191]. 